\title{
TRISOMY 21 IN A CHILD DUE TO PATERNAL NONDISJUNCTION AS DETERMINED BY RFA TECHNIQUE ${ }^{1}$
}

\author{
Ram S. Verma* and Harvey Dosik*** \\ *Division of Hematology and Cytogenetics The Jewish Hospital \\ and Medical Center Brooklyn, New York \\ **Department of Medicine State University of New York \\ Downstate Medical Center Brooklyn, New York
}

\begin{abstract}
Summary Sequential QFQ and RFA techniques were performed on a patient with Down syndrome and his parents. The paternal nondisjunctional event occurred in the first meiotic division. The origin of the extra chromosome 21 was determined by RFA. QFQ was not informative due to the absence of markers on chromosome 21. For maximal characterization of variations involving chromosome 21, sequential QFQ and RFA techniques are necessary. Consequently, the sequential QFQ and RFA techniques would be a definite asset in determining the source of the extra chromosome in patients with Down syndrome.
\end{abstract}

\section{INTRODUCTION}

Several cases of Down syndrome describing the parental origin of the extra chromosome 21 have been reported (Licznerski and Lindsten, 1972; Mutton, 1973; Robinson, 1973; Sasaki and Hara, 1973; Smith and Sachdeva, 1973; Uchida, 1973). In these reports, the origin of the extra chromosome 21 was based on the QFQ (Q-bands by fluorescence using quinacrine as suggested in the supplement (1975) of Paris Conference (1971)) polymorphisms (variations). Employing QFQ has certain limitations since the frequency of QFQ polymorphisms for chromosomes 21 is extremely rare $(1-25 \%$; Geralds and Pearson, 1974; Lin et al., 1976; McKenzie and Lubs, 1975), consequently, the origin of the extra chromosome 21 in Down syndrome can be determined in only a few cases (Mikkelsen et al., 1976).

Recently, we have reported that the short arm of human acrocentric chromosomes is polymorphic in colors as well as in sizes by RFA (R-bands by fluorescence using acridine orange) (Verma and Lubs 1975; Verma et al., 1976). Variations were classified into 1 of 6 colors, and into 1 of 5 sizes. Color and size variations are inherited in a Mendelian manner. Furthermore, there is no interrelationship

\footnotetext{
${ }^{1}$ Supported by the National Cancer Institute Contract \#1 CP43251

Received August 18, 1977
} 
between QFQ and RFA variations (Verma and Lubs, 1976). RFA would appear to be very useful in studying the origin of the extra chromosome 21 in Down syndrome, since more than $50 \%$ of the chromosome 21 's are polymorphic by this technique (Verma et al., 1977a, 1977b). The aim of this report is to illustrate a situation in which the origin of the extra chromosome 21 could not be determined by QFQ but could be detected by RFA.

\section{MATERIAL AND METHODS}

A boy with the classical features of Down syndrome is the third child of a 29 year old mother and a 32 year old father. Two other children, 7 and 5 are normal. The parents have no significant past medical history. Short-term lymphocyte cultures were harvested and chromosome preparations were made by standard techniques on the porband and parents. Sequential QFQ and RFA were performed (Verma and Lubs, 1975; 1976, Verma and Dosik, 1976). At least 25 metaphases were examined from each family member. QFQ chromosomes were photographed on Tri-X pan film using a Zeiss photomicroscope II and RFA chromosomes were photographed on Kodachrome 64 color film.

The present report describes the QFQ and RFA intensity variation in the short arms of the human acrocentric chromosomes. The five QFQ intensity levels established at the Paris Conference (1971) and a similar system reported in a recent study of McKenzie and Lubs (1975) were used to record the variable fluorescent intensities in specific chromosome regions, using a Code 1 for no fluorescence, 2 for pale fluorescence (as distal 1p), 3 for medium fluorescence (as the major bands of $9 q$ ), 4 ro intense fluorescence (as distal 13q) and 5 for brilliant fluorescence (as distal $\mathrm{Yq}$ ). Cells were printed on $5 \times 7$ photorite paper \#4. Scoring of intensity levels were performed without the knowledge of corresponding RFA variants.

The criteria for describing the colors and sizes of $D$ and $G$ short arms by RFA have been described earlier (Verma and Lubs, 1975). Color levels 1, 2, 3, 4, 5 and 6 represented red, red-orange, orange-yellow, pale yellow, bright yellow and pale green respectively. Size levels 1, 2, 3, 4, and 5 represented very small, small, average, large and very large respectively. At least 5-6 of the best differentiated cell from each individual were utilized for recording the RFA variants. RFA variants were scored without the knowledge of QFQ variants. After QFQ and RFA variants were determined the interrelationship between both methods was recorded on a two-dimensional data sheet.

\section{RESULTS AND COMMENTS}

The chromosomal constitution of the proband was $47, \mathrm{XY},+21$ in every cell. The father and mother were chromosomally normal. The QFQ intensities and RFA color and size levels recorded from each individual are shown in Fig. 1. There are 
Mother

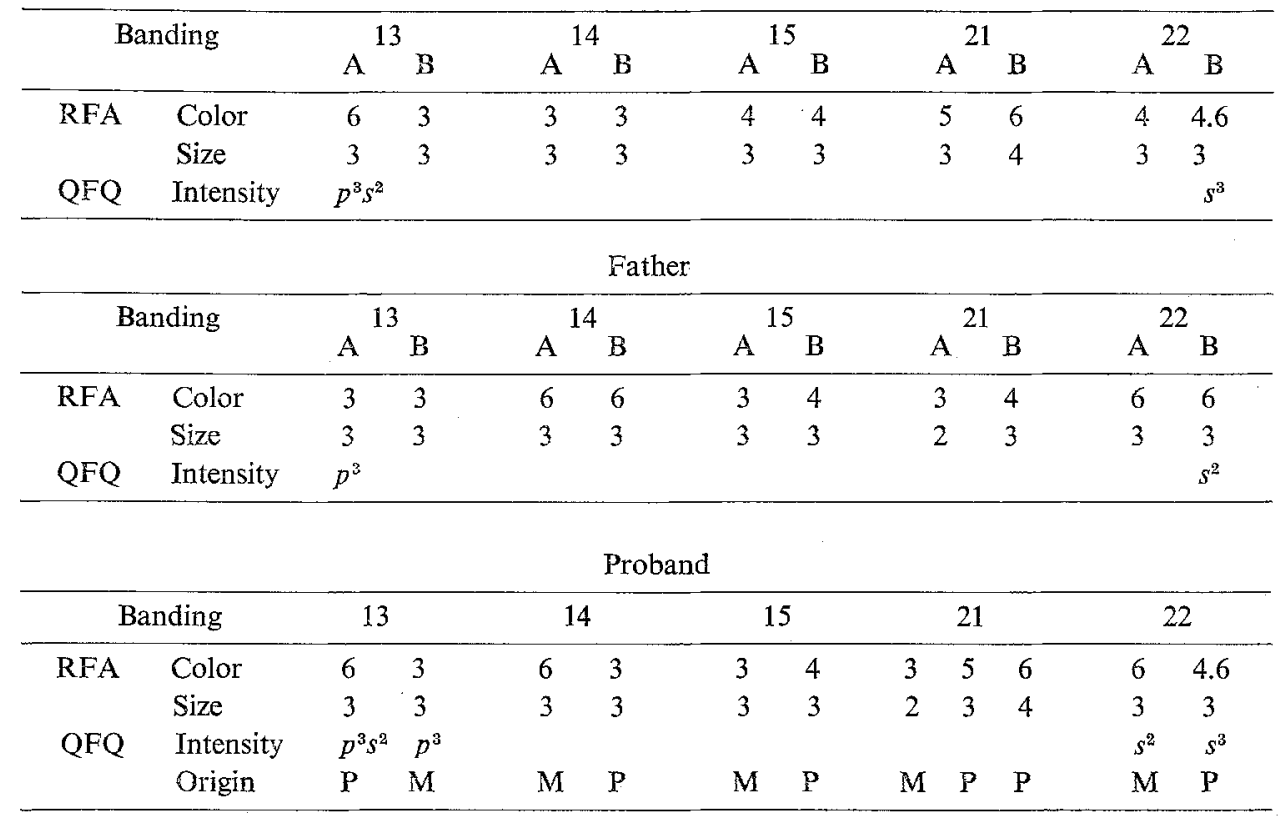

Fig. 1. Inheritance of $Q F Q$ and RFA polymorphisms in father, mother and proband. For RFA color and size levels, see Verma and Lubs (1975a). A, B=different homologs of same chromosome; $\mathrm{p}=$ band $\mathrm{p} 11 ; \mathrm{s}=$ satellite (band $\mathrm{p} 13$ ); $\mathrm{P}=$ paternal; $\mathrm{M}=$ Maternal.

no QFQ polymorphisms on chromosome 21 in either parent, consequently, using only QFQ we would have been unable to detect the parental origin of trisomy 21 (Fig. 1). RFA color polymorphisms were recorded from color slides as described earlier (Verma and Lubs, 1975). Since color reproduction in Journals is both difficult and expensive, only actual levels which were recorded are presented in Fig. 1. The color transparencies showing several color variations in $D$ and $G$ short arms will be provided on request. The polymorphisms were recorded on all acrocentric chromosomes to confirm the paternity of the probands. The father has two different chromosome 21's by RFA technique and both were inherited by the proband, indicating that nondisjunction occurred during the first paternal meiotic division. One of the maternal 21 chromosomes has a small short arm (level 2). This chromosome was inherited by the child. There are two reported cases of Down syndrome caused by maternal second meiotic nondisjunction, in which the maternal chromosome involved was a Gp- (de Grouchy, 1970; Juberg and Jones, 1970). Gp- is a size polymorphism and should be referred to as small size. These authors indicated that this was an abnormal chromosome and might have had an effect on the nondisjunctional event. Although this chromosome was present in mother and child, in our case the nondisjunctional event took place in the father. 
Previous studies using QFQ have determined the source of the extra chromosome 21 in less than $50 \%$ of cases. These studies have indicated a higher percentage of paternal nondisjunction than had been thought previously (Bott et al., 1975). These use of sequential QFQ and RFA should provide a much higher detetion rate and should be utilized in any large studies used to determine the source of the extra chromosome 21. If enough such data can be accummulated the recurring risk might be different depending on whether the non-disjunction was paternal or maternal in origin. This is turn might permit more precise counseling, although this is not currently established.

\section{REFERENCES}

Bott, C. E., Sekhon, G. S. and Lubs H. A. 1975. Unexpected high frequency of paternal origin of trisomy 21: (abst). 27th Annual meeting of the American Society of Human Genetics.

deGrouchy, J. 1970. 21p- maternel en couble exemplaire chez un trisomique 21. Ann. Genet 13: $52-55$.

Geralds, J.P.M. and Pearson, P. L. 1974. Fluorescent chromosome polymorphisms: frequencies and segregations in a Dutch population. Clin. Genet. 6: 245-257.

Juberg, R. C. and Jones, B. 1970. The Christchurch chromosome: mongolism, erythroleukemia and an inherited Gp- chromosome (Christchurch). New Eng. J. Med. 282: 292.

Licznerski, G. and Lindsten, J. 1972. Trisomy 21 in man due to maternal nondisjunction during the first meiotic division. Hereditas 70: 153-154.

Lin, C. C., Gedeon, M. M., Griffith, P., Smink, W. K., Newton, D. R., Wilkie, L. and Sewell, L. M. 1976. Chromosome analysis on 930 consecutive newborn children using quinacrine fluorescent banding technique. Hum. Genet. 31: 315-328.

McKenzie, W. H. and Lubs, H. A. 1975. Human Q and C chromosomal variations: distribution and incidence. Cytogenet. Cell Genet. 14: 97-115.

Mikkelsen, M., Hallberg, A. and Poulsen, H. 1976. Maternal and Paternal origin of extra chromosome in trisomy 21. Hum. Genet. 32: 17-21.

Mutton, D. E. 1973. Origin of extra chromosome in trisomy 21. Lancet I: 375.

Paris Conference: Standardization in Human Cytogenetics, Birth Defects: Original article series VIII: The National Foundation, New York (1971).

Paris Conference (1971), Supplement (1975): Standardization in Human Cytogenetics, Birth Defects: Original Articles Series, XI, 9 (1975).

Robinson, J. A. 1973. Origin of extra chromosome in trisomy 21. Lancet I: 131-133.

Sasaki, M. and Hara, Y. 1973. Paternal origin of the extra chromosome in Down's syndrome. Lancet II: $1257-1258$.

Smith, G. F. and Sachdeva, S. 1973. Origin of extra chromosome in trisomy 21. Lancet I: 487.

Uchida, I. A. 1973. Paternal origin of the extra chromosome in Down's syndrome. Lancet II:

Verma, R. S. and Lubs, H. A. 1975a. Variation in human acrocentric chromosomes with acridine orange reverse banding. Humangenetik 30: 225-235.

Verma, R. S. and Lubs, H. A. 1975b. A simple R banding technic. Amer. J. Hum. Genet. 27: 110-117.

Verma, R. S., Dosik, H. and Lubs, H. A. 1976. Frequencies of R polymorphisms in human acrocentric chromosomes with acridine orange reverse banding (RFA) in Caucasians (abs.) Excerpta Medica, Intl. Congress Sr. \#397.

Verma, R. S. and Lubs, H. A. 1976a. Inheritance of acridine orange $R$ variants in human acrocentric chromosomes. Hum. Hered. 26: 315-318. 
Verma, R. S. and Lubs, H. A. 1976b. Additional observations on the preparation of R banded chromosomes with acridine orange. Can. J. Genet. Cytol. 18: 45-50.

Verma, R. S. and Dosik, H. 1976. An improved method for photographing fluorescent human chromosomes. J. Microscopy 108: 339-341.

Verma, R. S., Dosik, H. and Lubs, H. A. 1977a. Frequency of RFA color polymorphisms of human acrocentric chromosomes in caucasians: Interrelationship with QFQ polymorphisms. Ann. Hum. Genet. (in press)

Verma, R. S., Dosik, H. and Lubs, H. A. 1977b. Size variation of the short arm of human acrocentric chromosomes determined by $\mathrm{R}$ banding by fluorescence using acridine orange (RFA). Hum. Genet. (in press) 